\title{
The Comparison of Image Retargeting Algorithms Based on Seam Carving
}

\author{
Zijuan Zhang \\ Shaanxi Polytechnic Institute, Xianyang, 712000, China
}

\begin{abstract}
All kinds of image retargeting algorithms based on seam carving are the representative types of discrete resizing methods. Seam carving algorithm refers to delete or insert pixel lines to resize image's technique. Pixel line pass through the whole image horizontally or vertically, deleting the pixel line makes the reduction, and copying the pixel line can make the magnification, processing level (vertical) pixel line can change image's height (width). In order to protect the main characteristic of image content, seam carving algorithms delete or insert pixel line which has the smallest energy every time. Various seam carving algorithms can scale the image's size, protect the important content of the source image, achieve good effect of adaptation, but this kind of algorithm will produce problem, such as excessive deleting distortion.
\end{abstract}

Keywords- seam carving; backward; forward; image retargeting

\section{INTRODUCTION}

On the Siggraph 2007, Avidan and Shamir [1] put forward the image retargeting method based on seam carving, this method is also known as Backward Seam Carving(BSC). Seam carving refers to the technology that removes or inserts pixel seam to scale image, this algorithm deletes or inserts the pixels line with minimum energy every time to realize image resizing which can protect the important content of the source image better and achieve the good effect about resizing result. With the BSC algorithm was put forward, some scholars propose many retargeting methods[2-4].

\section{IMAGE RETARGETING ALGORITHMS BASED ON SEAM CARVING}

In the literature [1], the content importance is calculated by gradient values of pixels. BSC algorithm is one of the most original seam carving algorithm. Figure 1 is a BSC algorithm example, figure 1 (a) has the original image, a horizontal and a vertical seam, pixels seam is a connected low energy path. Figure 1 (b) uses the gradient map to represent the energy of the original image(also known as the important degree map). Figure 1 (c) (d) are the vertical, horizontal path graph which is presented respectively to calculate pixels seam. BSC algorithm deletes horizontal pixel seam or inserts into the vertical pixel automatically to implement image retargeting, figure 1 (e) is the results of the original image by BSC algorithm. Figure 1 (f) is the result by using unified scale (Scaling, SCL). Compared with the traditional uniform scaling method, BSC algorithm achieves image retargeting and protects the important content of the source image better.

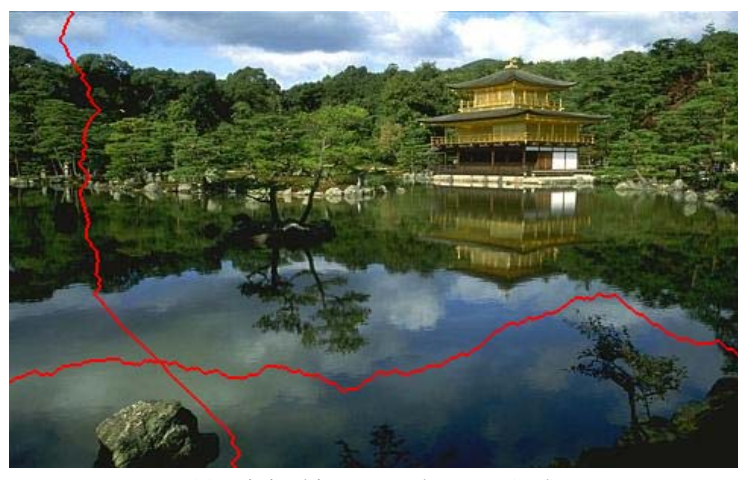

(a) original image and seams (red)

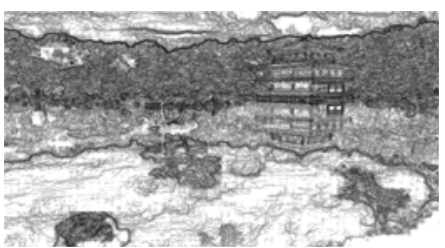

(b) gradient map

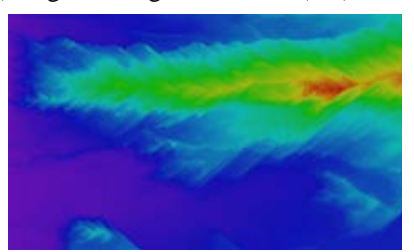

(c) vertical path

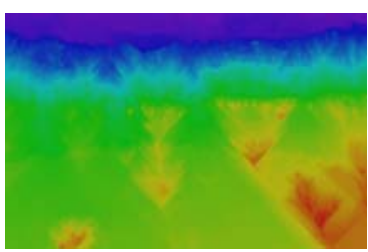

(d) horizontal path 


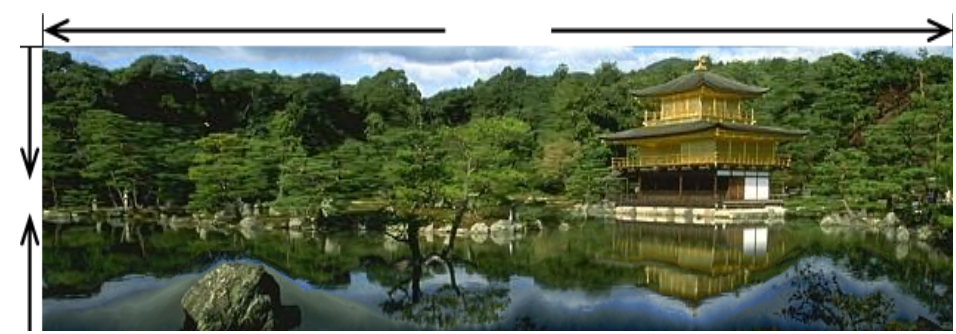

(e) the retargeting result by BSC

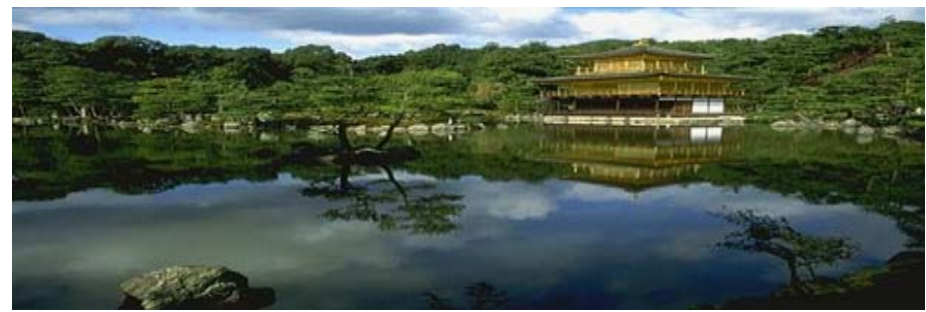

(f) the resizing result by SCL

FIGURE I. THE EXAMPLE OF BSC ALGORITHM[1].

In order to define the importance degree of pixels in the image, in the original literature BSC algorithm defines image gradient norm as the energy function. Supposing the original image is $I$, then the energy function is

$$
e(I)=\left|\frac{\partial}{\partial x} I\right|+\left|\frac{\partial}{\partial y} I\right|
$$

The value of the energy function represents the importance degree of the pixels. Supposing the size of image $I$ is $m \times n$, $m$ is column, $n$ is row. Then the vertical pixels seam $s^{v}$ can be defined as

$$
s^{v}=\left\{s_{i}^{v}\right\}_{i=1}^{n}=\{(x(i), i)\}_{i=1}^{n}, \text { s.t. } \forall i,|x(i)-x(i-1)| \leq 1
$$

$x(i)$ is mapping: $x:[1, \cdots, n] \rightarrow[1, \cdots m]$. That is to say, vertical pixel line is a 8 connected pixels path from the first line to the last line in the image, and every line in the source image contains only one pixel in each line. If $y$ is mapping: $y:[1, \cdots m] \rightarrow[1, \cdots, n]$, then the horizontal seam line $s^{h}$ can be defined as

$$
s^{h}=\left\{s_{j}^{h}\right\}_{j=1}^{m}=\{(j, y(j))\}_{j=1}^{m}, s . t, \forall j,|y(j)-y(j-1)| \leq 1
$$

The pixels on vertical seam $s$ is

$$
I_{s}=\left\{I\left(s_{i}\right)\right\}_{i=1}^{n}=\{I(x(i), i)\}_{i=1}^{n}
$$

It is important to note that when vertical (horizontal) seam is removed from the source image, pixels on the seam right side should move to left , to make up for the gap due to deleting.

Based on energy function, a cost of vertical seam line $E(s)$ can be defined as

$$
E(s)=E\left(I_{s}\right)=\sum_{i=1}^{n} e\left(I\left(s_{i}\right)\right)
$$

Minimizing seam cost to look for the optimal seam $s^{*}$

$$
s^{*}=\min _{s} E(s)=\min _{s} \sum_{i=1}^{n} e\left(I\left(s_{i}\right)\right)
$$

We could use dynamic program algorithm to search for the optimal seam. For each position $(i, j)$ about every pixel on image, the cumulative energy $M(i, j)$ is

$$
M(i, j)=e(i, j)+\min (M(i-1, j-1), M(i, j-1), M(i+1, j-1))
$$

In order to find the optimal perpendicular path, we traverse all pixels from the second line to the last line in the image firstly and calculate the accumulated energy. At the end of this process, the accumulated energy minimum point in last line is the optimal vertical line in the end. Then from that point back, looking for the cumulative minimum energy pixel location and will continue to back to this place as the new starting point, continuing to look for it in the same method, repeating this iterative process until the first line of the image to find the optimal path pixel vertical line. The definition of the optimal level pixel line path is also a similar process. 
Literature [5] has noticed that BSC algorithm only considers energy of the pixel itself, put forward seam carving(FSC) method. This algorithm increases that due to deleting the pixel which makes the new adjacent pixels, implements improvement on BSC algorithm. Because the FSC algorithm considers the deleting pixel seam that makes other adjacent pixel to produce new relations and increase energy, compared to the method of BSC, FSC algorithm can get better result. In addition, the literature [6-7] propose different improved seam carving algorithms.

\section{CONCLUSION}

Although the appearance of many new scaling methods based on BSC algorithm which obtain the good effect of scaling, as the original seam carving method, the status of the BSC algorithm is without a doubt, its pioneer and innovation is unmatched by other methods. At the same time in a variety of improved algorithms based on BSC method, the effect of forward seam carving algorithm (FSC) is significant and can effectively eliminate artificial noise points. Whether BSC, FSC or other improved algorithm based on seam carving, they are discrete method which is based on pixel level which will produce distortion problem, such as excessive deleting distortion.

\section{REFERENCES}

[1] S. Avidan and A. Shamir, "Seam carving for content-aware image resizing,” in ACM Transactions on Graphics, 2007,vol. 26(3), pp. 1-10

[2] J.C. Yoon, S.Y. Lee, and I.K. Lee, “Optimized image resizing using flow-guided seam carving and an interactive genetic algorithm," in Multimedia Tools and Applications, 2014, vol. 71(3), pp. 1013-1031

[3] M. Kazu, and I. Masaaki, "Seam merging for image resizing with structure preservation,” in 2011 IEEE International Conference on Acoustics, Speech and Signal Processing, 2011, pp. 1001-1004

[4] J.D. Wei, Y.J. Lin and Wu Y.J, "A patch analysis method to detect seam carved images,” in Pattern Recognition Letters, 2014, vol. 36, pp. 100106

[5] M. Rubinstein, A. Shamir and S. Avidan, "Improved seam carving for video retargeting," in ACM Transactions on Graphics, 2008, vol. 27(3), pp. 1-9

[6] H. Nicholas and E. Parisa, "Seam carving for enhancing image usability on mobiles," in Proceedings of the 22nd British HCI Group Annual Conference on HCI, 2008, pp. 131-134

[7] N. Ryo, U. Kei and T. Keita "Stereo image retargeting with shift-map," in IEICE Transactions, 2011, vol. 94-D(6), pp. 1345-1348 